\title{
AN INTRODUCTION TO THE FIBEROPTIC LARYNGOSCOPE
}

\author{
ThOMAS H. WITTON
}

\begin{abstract}
The fiberoptic laryngoscope can make difficult or impossible tracheal intubations as easy as a routine procedure. Although this instrument has been available for many years it has never achieved the popularity it deserves because anaesthetists have abandoned it after a few unsuccessful attempts to use it.

Detailed procedure for the successful use of this instrument and the reasons for failure are described. Facility with the instrument should be developed by practice with patients where no difficulty in intubation is anticipated rather than making first attempts when problems arise.
\end{abstract}

Key Words: Equipment, Fiberoptic Laryngoscope.

AT ONE TIME or another, every anaesthetist has been faced with the problem of a difficult intubation caused by such things as stiff neck, anterior larynx, inability to open the mouth fully, or the presence of prominent or fragile teeth. Usually the intubation is accomplished with a little persistence and a lot of luck and the problem is soon forgotten. Only rarely is the operation cancelled because of total inability to intubate the trachea. There has been an instrument on the market for many years which makes these difficult or impossible intubations as easy and sure as a routine procedure. Unfortunately, the fiberoptic laryngoscope has never achieved the popularity it deserves, and in talking with other anaesthetists, the story is something like "I tried it a couple of times, but couldn't see anything with the damn thing, so we sent it back," or "It looks great when you try it on the mannequins but it's sure no good for real people."

The problem stems from the fact that when one purchases the instrument that is all that one buys, and no instructions are included with it. This paper will attempt to provide guidelines for the beginner; as he becomes more proficient each anaesthetist will devise his own set of rules.

\section{The Equipment}

The fiberoptic laryngoscope consists of light source, handle, body and the flexible laryngoscope "blade". On the right hand side of the body is a small knob which controls the movement of the distal end of the blade. This movement is in

Thomas H. Witton, M.D., Dip.Anaes., F.R.C.P.(C), Department of Anaesthesia, Joseph Brant Memorial Hospital, Burlington, Ontario.

Address for Correspondence: 906-1363 Lakeshore Road, Burlington, Ontario L7S IB2. one plane only; that is, the tip may be flexed either anteriorly or posteriorly. There is no lateral movement. If lateral movement is desired, it is done by rotating the whole instrument.

The light source may be powered by batteries or by an external source. The scope comes with batteries which fit into the handle, but there is some advantage in buying an external light source and not wasting time with the batteries. Actually it might not be necessary to purchase a light source, because one can use any light source in the hospital, such as those available for cystoscopes, laparoscopes and bronchoscopes; only a cord will be needed that fits whichever light source one decides to use.

\section{Preparation}

The eyepiece of the laryngoscope rotates, which serves to focus. Looking through the eyepiece, one holds the distal end of the scope about $2-3 \mathrm{~cm}$ from some detailed object such as writing, and rotates the eyepiece until the writing is sharply focussed. It might be advisable to fasten a small piece of adhesive around the eyepiece to keep it from moving when the focus is satisfactory.

The light source is now turned on. If the bulb is going to burn out it will do so when it is turned on and not after the patient is asleep and paralyzed.

The next step is to select the proper tracheal tube, cut to the proper length in the usual fashion, and between 7.5 and $8.5 \mathrm{~mm}(32-36 \mathrm{Fr})$ in diameter. Smaller tubes do not fit readily over the laryngoscope and tubes larger than this leave too large a gap between the laryngoscope and the tube, allowing the tube to become caught on the arytenoids. A small amount of lubricant is put on the outside of the tube, and also some on the

Canad. Anaesth. Soc. J., vol. 28, no. 5, September 1981 


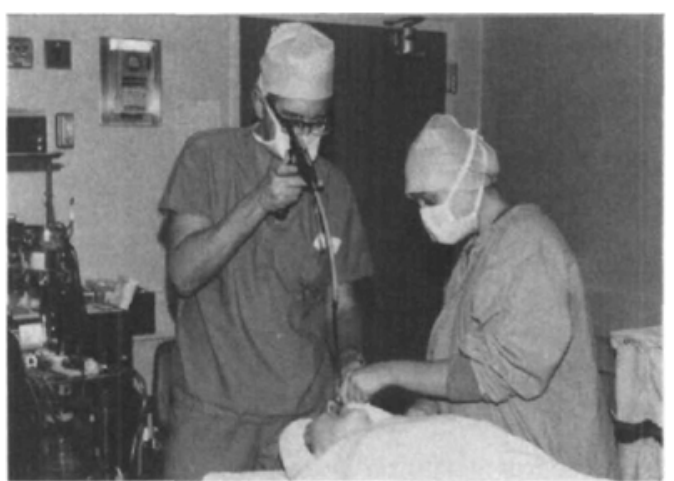

Figure 1 Tracheal tube correctly mounted. Assistant holding tongue forward. Right hand holding laryngoscope with thumb and index finger controlling directing knob, left hand keeping head extended and thumb and index finger directing laryngoscope in midline.

laryngoscope, being careful not to get any onto the lens. The tube is then passed all the way up the laryngoscope, until it abuts the body, leaving the distal 6-12 cm exposed and freely flexible (Figure 1). The tracheal tube must not extend over the distal end of the instrument in the expectation of bending the tube with the laryngoscope. The tube will not bend and one succeeds only in breaking the instrument itself.

Any induction agent can be used as long as complete relaxation is accomplished. Probably thiopentone and succinylcholine are the most commonly used agents, and these are perfectly satisfactory. It is a wise precaution to have a duplicate dosage drawn up in syringes, because when one is first learning it is very tempting to be "just a few more seconds", and then one suddenly realizes that the patient needs ventilating immediately, but needs more relaxation first. The availability of a second set of agents will overcome this problem. One should not rely on nurses to refill syringes quickly, under these circumstances.

\section{Intubation}

After induction the head is put into the usual position for intubation; that is the neck is flexed and the head extended at the atlanto-occipital joint.

The most important secret to success is to have an air space at the end of the laryngoscope. Just as a bladder is distended for cystoscopy, and the abdomen distended for laparoscopy, so for laryngoscopy it is essential also that the end of the laryngoscope be kept away from tissue. This is most readily accomplished by having an assistant pull the tongue forward, away from the palate and posterior pharyngeal wall.

With the laryngoscope in the right hand, the left middle and ring fingers are placed behind the patient's upper teeth and, using these fingers to keep the head extended, the fiberoptic is guided with the thumb and index finger, being sure to keep it exactly in the mid-line (Figures 1 and 2). As the right hand is lowered and the laryngoscope is guided with the left finger and thumb the surface of the tongue is seen gliding past the lens, and then the uvula will come into view (Figure 3). As the scope nears the posterior part of the tongue, using the fingers of the right hand, the knob is turned to give a slight amount of flexion to the scope. As one descends further, the epiglottis will come into view.

Occasionally the anterior surface of the epiglottis is seen, but usually it is the free edge that comes into view first (Figures $4 \& 5$ ). The epiglottis is distinctly paler than the surrounding structures, being almost yellow in colour. Getting past the epiglottis is the only hard part of the procedure. If it is small, it will be standing up, and the free edges will be seen first. In this case, simply advancing the scope will allow one to pass the epiglottis. In patients with a large epiglottis the procedure is a little more difficult. This will become apparent when the first part of the epiglottis to be seen is the anterior surface, because it is large and lying back over the glottis. At this point one must make sure that the tongue is as far forward as possible, and then the laryngoscope is extended backwards with the knob to pass around the free edge of the epiglottis, which will be quite far posteriorly. Usually this has to be done blind, as the instrument will come to lie against the epiglottis and as soon as this happens nothing more can be seen. With a little practice, one soon learns how far to intro-

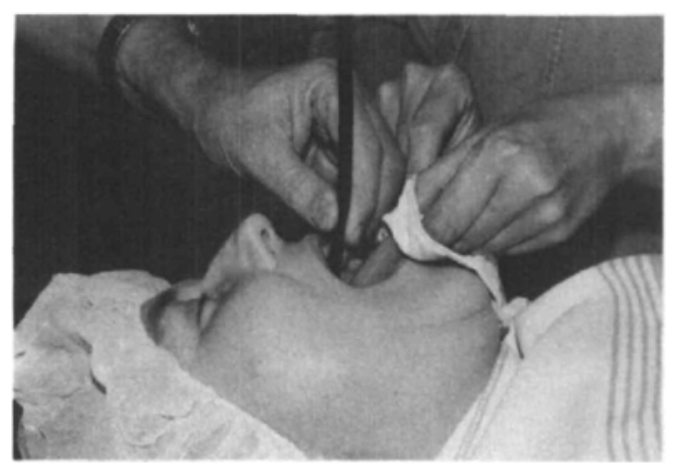

Figure 2 Detailed view of Figure 1. 


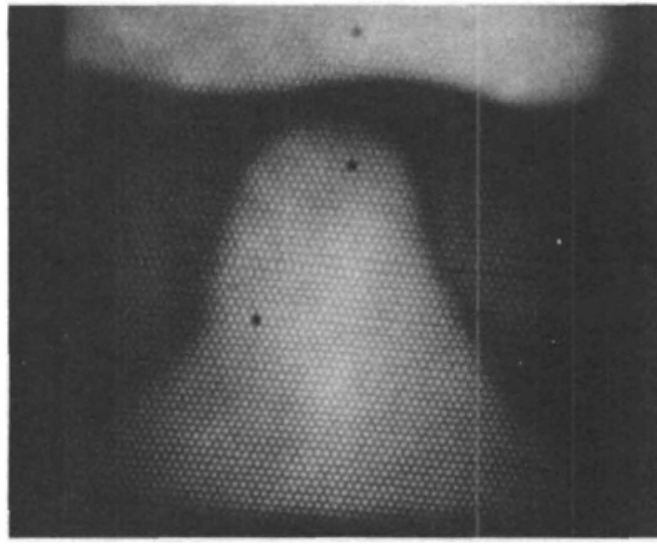

FIGURE 3 Upper surface of tongue and uvula.

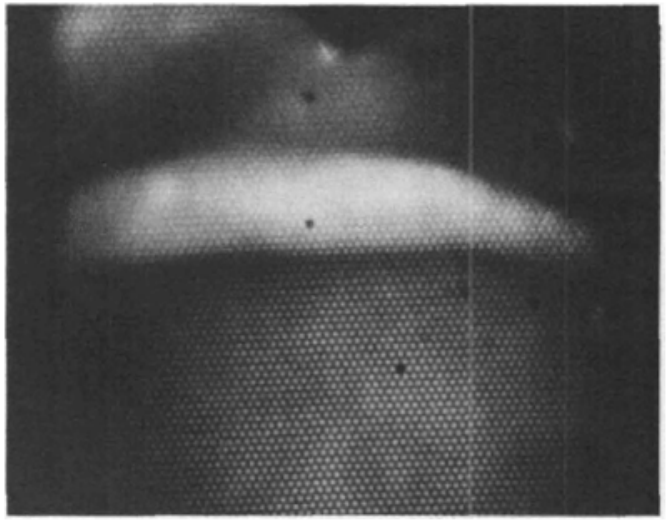

FIGURE 4 Free edge of epiglottis.

duce the laryngoscope blind and then to flex it again to bring the glottis into view. If at any time one becomes lost and cannot see anything but a mass of pink, it is probably because the laryngoscope is no longer in the mid-line. The best procedure is to withdraw, re-establish the scope in the mid-line and start over again.

After the cords come into view (Figures 6 and 7), one simply advances the instrument using the knob to steer it between the cords. As the cords are touched one will once again be unable to see anything and this is the reason why complete relaxation is so essential. If the cords are not totally relaxed they will adduct and deflect the laryngoscope into the oesophagus.

The view of the inside of the trachea is unique and, once seen, cannot possibly be confused with anything else. All the tracheal rings are clearly visible (Figure 8) and, by advancing the laryngoscope a few centimetres, the carina can be identified.

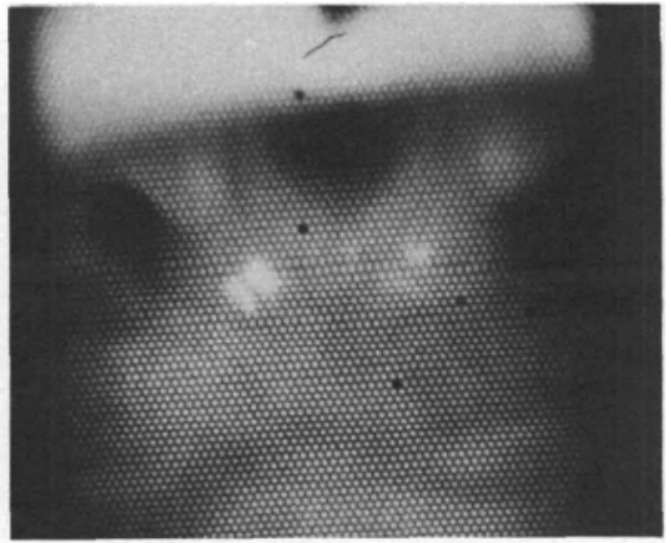

FIGURE 5 Epiglottis, arytenoid cartilages and posterior half of glottis.

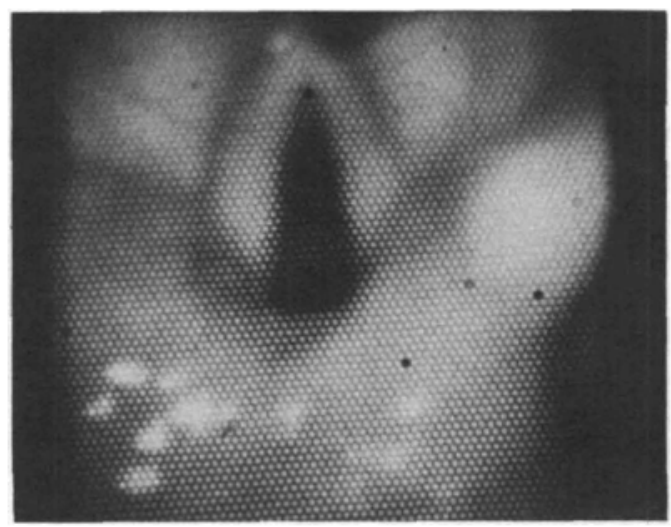

FIGURE 6 Glottis, with arytenoids and cords.

Once the laryngoscope is inside the trachea it is advanced for about $6 \mathrm{~cm}$ and then, holding it firmly in the right hand, the tracheal tube is advanced with the left hand until it is in the trachea. The laryngoscope is then withdrawn, the connector inserted into the tube, and the anaesthesia carried on in the usual way. Usually the tube slides into the trachea quite easily, but sometimes the tip will become caught behind the arytenoids. If this happens, the laryngoscope should be given to an assistant to hold; one then reaches into the mouth with the index finger of the right hand and eases the tube forward as it is slid gently down with the left hand. Using a tube larger than $8.5 \mathrm{~mm}$ tends to make this happen more often.

If nasal intubation is desired exactly the same procedure can be followed, except that one will now see the turbinates rather than the tongue. Great care must be taken not to cause bleeding, as even one drop of blood on the lens makes 


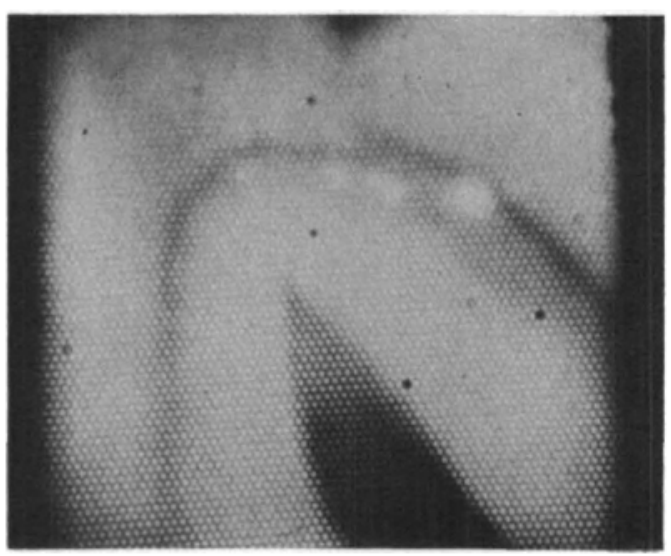

FiguRe 7 Vocal cords, just before laryngoscope enters the trachea.

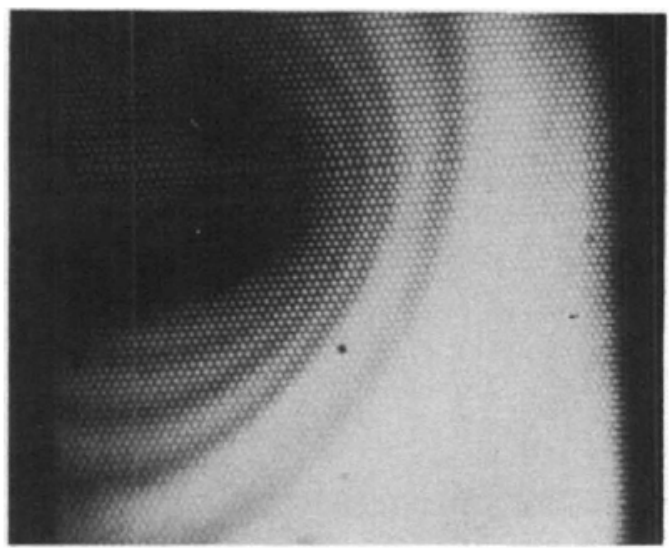

FIGURE 8 Inside of trachea showing cartilaginous rings. vision impossible. For this reason, if one has a patient who would appear to present a potential intubation problem, it is wise to start right off with the fiberoptic laryngoscope, or at least to switch to it early, before any bleeding has been caused.

\section{Care of the Instrument}

Cleaning of the fiberoptic laryngoscope is done easily with warm water. Avoid getting anything wet except the fiberoptic part. Drying must be done gently, otherwise the covering of the instrument might all be pulled down to its distal extremity. We do not autoclave or gas sterilize our instrument and, while this may alarm some people, one can say in our defense that we have веen using one for over five years and never, to our knowledge, have we had a problem because of this technique.

\section{Summary}

Like any medical technique, the more it is practised the better one becomes at it. The instrument should be used for all normal intubations until one becomes adept at it. There is no sense waiting until one has a difficult intubation and then expecting success without having practised beforehand. In summary, the main points to remember are:

Two sets of induction agents should be drawn up.

The Head must be in proper position.

The Tongue must be held forward.

The instrument must be kept in the midline.

The right index finger might be required to free the tracheal tube from the arytenoids.

\section{RÉSUMÉ}

Le laryngoscope à fibres-optiques peut transformer une intubation difficile ou impossible en geste aussi facile qu'une laryngoscopie de routine. Bien que cet instrument ait été disponible depuis plusieurs années il n'a jamais atteint la popularité qu'il mérite car beaucoup d'anesthésistes l'abondonnent après quelques essais infructueux.

Cet article décrit en détail la technique d'utilisation de l'appareil et discute des causes d'échec. On devrait se familiariser avec l'usage de l'instrument chez des patients où une intubation facile est prévue plutôt que d'attendre un cas difficile pour apprendre à s'en servir. 